\title{
Complementary actions of melatonin on angiogenic factors, the angiopoietin/Tie2 axis and VEGF, in co-cultures of human endothelial and breast cancer cells
}

\author{
ALICIA GONZÁLEZ-GONZÁLEZ, ALICIA GONZÁLEZ, CAROLINA ALONSO-GONZÁLEZ, \\ JAVIER MENÉNDEZ-MENÉNDEZ, CARLOS MARTÍNEZ-CAMPA and SAMUEL COS \\ Department of Physiology and Pharmacology, School of Medicine, University of Cantabria and \\ Instituto de Investigación Sanitaria Valdecilla (IDIVAL), 39011 Santander, Spain
}

Received May 24, 2017; Accepted September 15, 2017

DOI: $10.3892 /$ or.2017.6070

\begin{abstract}
Melatonin exerts oncostatic activity in breast cancer through antiangiogenic actions. There, the aim of the present study was to ascertain whether melatonin modulates, in a coordinated action, angiopoietin-1 (ANG-1), ANG-2, their cognate Tie2 receptor and VEGF in co-cultures of human endothelial cells (HUVECs) and breast cancer (MCF-7) cells. To accomplish this we used co-cultures of human breast cancer cells (MCF-7) or non-malignant human mammary epithelial cells (MCF-10A) with endothelial cells (HUVECs). The presence of breast cancer cells increased HUVEC proliferation and $1 \mathrm{mM}$ melatonin prevented this effect. ANG-1, ANG-2 and VEGF levels in co-culture media and mRNA expression were upregulated and Tie 2 mRNA expression was downregulated in the HUVECs and MCF-7. Melatonin (1 mM) downregulated ANG-1, ANG-2 and VEGF levels in the co-culture media and mRNA expression in both types of cells and upregulated Tie2 mRNA expression in HUVECs. ANG-1, ANG-2, Tie2 and VEGF mRNA expression were not modified during HUVEC/MCF-10A co-culture. Estradiol (10 nM) increased ANG-1, ANG-2 and VEGF mRNA expression in HUVECs and melatonin $(1 \mathrm{mM})$ counteracted this effect. We conclude that melatonin simultaneously coordinates downregulation of angiopoietins with a reduction in VEGF, which could be an effective therapeutic strategy for blocking tumor angiogenesis.
\end{abstract}

\section{Introduction}

The formation of new blood vessels from the existing vasculature is essential to support tumoral development (1). Since

Correspondence to: Dr Samuel Cos, Departamento de Fisiología y Farmacología, Facultad de Medicina, Universidad de Cantabria, Cardenal Herrera Oria s/n, 39011 Santander, Spain

E-mail: coss@unican.es

Key words: melatonin, endothelial cells, breast cancer, angiopoietins, HUVEC, VEGF neo-angiogenesis is a key event in tumor progression, antiangiogenic agents are considered as an alternative strategy in cancer treatment (2). In healthy tissues, the balance of angiogenic activating and inhibiting factors determines the transition of endothelial cells between a pro-angiogenic or a quiescent stage (2). Vascular endothelial growth factor (VEGF) and angiopoietins are some of the main pro-angiogenic factors $(3,4)$. VEGF is one of the most important molecules stimulating tumoral angiogenesis and an increased VEGF expression has been described in different types of cancers, such as breast, brain, lung, urothelial and gastrointestinal tract tumors (5). In mammary tumors, VEGF is released by human breast cancer cells and binds VEGF receptors triggering proliferation, growth, survival and migration of endothelial cells (6-8). Angiopoietins are endothelial-produced proteins which bind the tyrosine kinase receptor Tie2, modulating vessel stability (9). Although four angiopoietins (ANG-1 to ANG-4) have been described, ANG-1 and ANG-2 are the most widely studied. ANG-1 is a Tie2 receptor agonist expressed in vascular mural cells and non-vascular normal and tumor cells. It is a vascular stabilizing factor that stimulates recruitment of pericytes and smooth muscle cells, collaborates to maintain vascular integrity and quiescence and is also able to promote angiogenesis $(9,10)$. Contrary to ANG-1, ANG-2 behaves as an antagonist of Tie2, blocking ANG-1-mediated phosphorylation of Tie 2 therefore reducing the interactions between endothelial and perivascular support cells and extracellular matrix, decreasing vascular integrity and causing vessel regression in the absence of angiogenic factors, whereas it potentiates angiogenesis in the presence of $\operatorname{VEGF}(9,11)$. ANG-2 is mainly produced by endothelial cells and formed during vascular remodelling (11). A wide number of malignant tumors show upregulation of both ANG-1 and ANG-2 angiopoietins, promoting a shift in the ANG-1:ANG-2 ratio towards ANG-2 which in the presence of VEGF is associated with tumor angiogenesis (12). Tie2 receptors bind directly to angiopoietins, have strong tyrosine kinase activity, and are selectively expressed in endothelial cells, although other cell types including early hematopoietic cells and subsets of monocytes also express Tie2 (13). Angiogenesis is dependent on a dynamic equilibrium between the production of VEGF and 
angiopoietins that must be both quantitatively and temporally coordinated (14).

Melatonin, synthesized and released from the pineal gland, has been demonstrated to have oncostatic actions in hormonedependent tumors (15-17). Melatonin exerts oncostatic activity through several biological mechanisms including: indirect effects of melatonin via the hypothalamic-pituitaryreproductive axis, which results in the downregulation of some of the hormones that may stimulate proliferation of malignant cells, such as estrogenic compounds produced by the gonads (18); direct antiestrogenic molecular mechanisms that take place inside epithelial cells of the mammary tissue $(19,20)$; antioxidant effects $(21)$; melatonin has been also implicated in both hemopoiesis and enhancement of anticancer immunity (22); inhibition of telomerase in epithelial malignant cells $(23,24)$; inhibition of fatty acid uptake and fat metabolic pathways $(25,26)$ and inhibition of angiogenesis (27-29). With respect to its antiangiogenic effects, in co-cultures of human breast cancer and endothelial cells, melatonin was found to regulate the tumor microenvironment through the downregulation of VEGF expression in human breast cancer cells, which results in a decrease of the secretion of VEGF and as a consequence, a reduction in the levels of VEGF around endothelial cells (28-30). Melatonin strongly inhibits the proliferation as well as the invasion/migration of endothelial cells, disrupts tube formation and counteracts the VEGF-stimulated tubular network assembly (29). Melatonin also shows indirect antiangiogenic effects by inhibiting various other tumor growth factors, such as IGF, EGF and ET-1, which are strong mitogens and stimulators of cancer angiogenesis (31). Neutralization of reactive oxygen species which, during hypoxia, plays an important role in stabilizing hypoxia-inducible factor HIF- $\alpha$ (32) is another indirect antiangiogenic effect of melatonin.

Although a variety of factors can modulate endothelial cell response, a complementary and coordinated action of VEGF and angiopoietins during angiogenesis is required $(9,33)$. Since melatonin can modulate VEGF in tumor cells and has antiangiogenic effects, in the present study, we aimed to ascertain whether melatonin modulates in a coordinated action angiopoietins 1 and 2, their cognate Tie 2 receptor and VEGF in vitro in endothelial cell cultures. To accomplish this we used co-cultures of human breast cancer cells (MCF-7) with human umbilical vein endothelial cells (HUVECs).

\section{Materials and methods}

Cells and culture conditions. Human umbilical vein endothelial cells (HUVECs) were purchased from the American Type Culture Collection (ATCC; Rockville, MD, USA). They were maintained as monolayer cultures in $58.1 \mathrm{~cm}^{2}$ plastic culture plates in Vascular Cell Basal Medium (VCBM) (ATCC) supplemented with Endothelial Cell Growth Kit-BBE (ATCC) which consisted of $2 \%$ fetal bovine serum (FBS; PAA Laboratories, Pasching, Austria), $0.2 \%$ bovine brain extract, $5 \mathrm{ng} / \mathrm{ml} \mathrm{rhEGF}$, $10 \mathrm{mM}$ L-glutamine, $0.75 \mathrm{U} / \mathrm{ml}$ heparin sulfate, $1 \mu \mathrm{g} / \mathrm{ml}$ hydrocortisone hemisuccinate, $50 \mu \mathrm{g} / \mathrm{ml}$ ascorbic acid, penicillin $(20 \mathrm{U} / \mathrm{ml})$ and streptomycin $(20 \mu \mathrm{g} / \mathrm{ml})$ (Sigma-Aldrich, Madrid, Spain) at $37^{\circ} \mathrm{C}$ in a humid atmosphere containing $5 \%$ $\mathrm{CO}_{2}$. To avoid genetic mutation and low viability, no more than

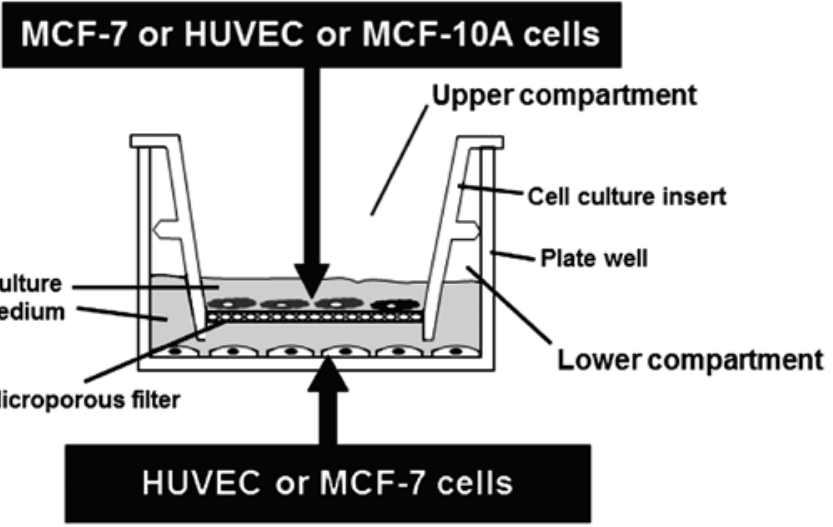

Figure 1. Schematic representation of the Falcon 6-multiwell plates and Falcon cell culture inserts used in the co-cultures. In some experiments HUVECs were seeded in the lower compartment and MCF-7 or MCF-10A cells in the upper compartment. In other experiments, the cells were exchanged with HUVECs at the top and MCF-7 or MCF-10A in the bottom.

six passages of HUVECs were used for the following experiments.

MCF-7 human breast cancer cells were purchased from ATTC. They were maintained as monolayer cultures in $58.1 \mathrm{~cm}^{2}$ plastic culture plates in Dulbecco's modified Eagle's medium (DMEM) (Sigma-Aldrich) supplemented with $10 \%$ FBS, penicillin $(20 \mathrm{U} / \mathrm{ml})$ and streptomycin $(20 \mu \mathrm{g} / \mathrm{ml})$ at $37^{\circ} \mathrm{C}$ in a humid atmosphere containing $5 \% \mathrm{CO}_{2}$.

Non-malignant human mammary epithelial cell line (MCF-10A) was purchased from ATTC. They were maintained as monolayer cultures in $58.1 \mathrm{~cm}^{2}$ plastic culture plates in DMEM/F12 supplemented with $5 \%$ horse serum, $0.5 \mu \mathrm{g} / \mathrm{ml}$ hydrocortisone (all from Sigma-Aldrich), $20 \mathrm{ng} / \mathrm{ml}$ epidermal growth factor (R\&D Systems Europe Ltd., Abingdon, $\mathrm{UK}$ ), $100 \mathrm{ng} / \mathrm{ml}$ cholera toxin, $10 \mu \mathrm{g} / \mathrm{ml}$ insulin (both from Sigma-Aldrich), penicillin $(20 \mathrm{U} / \mathrm{ml})$ and streptomycin $(20 \mu \mathrm{g} / \mathrm{ml})$ at $37^{\circ} \mathrm{C}$ in a humid atmosphere containing $5 \% \mathrm{CO}_{2}$.

Co-culture of HUVECs and MCF-7 or MCF-10A cells. HUVECs were co-cultured together with MCF-7 or MCF-10A cells using Falcon 6-multiwell plates and Falcon cell culture inserts. HUVECs were plated $\left(50 \times 10^{4}\right.$ cells/well) on the bottom wells in VCBM supplemented with $2 \%$ FBS and incubated overnight. At this time, MCF-7 cells (40x10 4 cells) or MCF-10A cells $\left(30 \times 10^{4}\right.$ cells) were seeded on the permeable membrane $(0.45-\mu \mathrm{m})$ of the tissue-culture inserts in DMEM supplemented with $10 \%$ FBS for $24 \mathrm{~h}$. HUVECs and MCF-7 or MCF-10A cells were cultured separately for $24 \mathrm{~h}$ to establish attachment. After $24 \mathrm{~h}, \mathrm{MCF}-7$ or MCF-10A seeded inserts were moved over the HUVEC cell cultures in the 6-well plates in fresh VCBM supplemented with $2 \%$ FBS to create the hanging co-culture setup (Fig. 1). Due to the membrane pore size and diffusional distance between cells within this setup, cell to cell contact is prevented but paracrine signalling can occur between endothelial cells in the 6-well plate and epithelial cells on the insert. After $24 \mathrm{~h}$, media were replaced with VCBM supplemented with $2 \%$ FBS containing melatonin ( $1 \mathrm{mM}$ or $1 \mathrm{nM}$ ) or vehicle (ethanol) for $4 \mathrm{~h}$ to measure mRNA expression of angiogenic factors or for $72 \mathrm{~h}$ to measure proliferation and ANG-1, ANG-2 and VEGF protein levels. At the 
end of the experiment, media were collected, centrifuged to remove particulates and subjected to measurement of ANG-1, ANG-2 and VEGF protein levels. Cells (HUVECs) in the bottom plate were evaluated for proliferative indices by the MTT method and for ANG-1, ANG-2, Tie2 and VEGF mRNA expression by RT-PCR. Since we were only able to measure ANG-1, ANG-2, Tie2 and VEGF mRNA expression of the cells that were in the lower compartment, in other experiments MCF-7 cells were plated $\left(80 \times 10^{4}\right.$ cells/well) on the bottom wells and HUVECs $\left(30 \times 10^{4}\right)$ on the permeable membrane of the tissue-culture inserts to be able to measure ANG-1, ANG-2, Tie2 and VEGF mRNA expression by RT-PCR in MCF-7 cells.

Measurement of cellular proliferation. Since the reduction of tetrazolium salts is widely accepted as a reliable way to examine cell proliferation, we used the 3-(4,5-dimethylthiazol-2-yl)2,5-diphenyltetrazolium bromide (MTT) method (34), reading absorbance at $570 \mathrm{~nm}$ in a microplate reader (Multiskan RC 351; LabSystems Vienna, VA, USA). MTT was obtained from Molecular Probes Inc. (Eugene, OR, USA).

Measurement of ANG-1, ANG-2, Tie2 and VEGF mRNA expression. Analysis of the ANG-1, ANG-2, Tie2 and VEGF mRNA expression in HUVECs, MCF-7 and MCF-10A cells was carried out by real-time reverse transcription RT-PCR after incubation of cells with either $1 \mathrm{mM}$ melatonin and/or estradiol $10 \mathrm{nM}$ (Sigma-Aldrich) and/or vehicle (ethanol) for $4 \mathrm{~h}$. The total cellular RNA was isolated from HUVECs or MCF-7 cells and purified using the NucleoSpin RNA II kit (Machenery-Nagel, Düren, Germany) following the manufacturer's instructions. Integrity of RNA was assessed by electrophoresis in ethidium bromide-stained 1\% agaroseTris-borate EDTA gels. The absorbance ratio A260 ${ }_{\mathrm{nm}} / \mathrm{A} 280_{\mathrm{nm}}$ was $>1.8$. For cDNA synthesis, $0.5 \mu \mathrm{g}$ of total RNA was denatured at $65^{\circ} \mathrm{C}$ for $10 \mathrm{~min}$ and reverse transcribed for 50 min at $45^{\circ} \mathrm{C}$ with a cDNA synthesis kit (BioLine, London, $\mathrm{UK}$ ) in a final volume of $20 \mu \mathrm{l}$ in the presence of $500 \mathrm{ng}$ of oligo(dT) $)_{12-18}$ primers. Quantitative real-time PCRs were performed using the following set of human ANG-1-specific primers: [5'-GAAGGGAACCGAGCCTATTC-3' (forward) and 5'-AGGGCACATTTGCACATACA-3' (reverse)]; ANG-2specific primers [5'-AAGAGAAAGATCAGCTACAGG-3' (forward) and 5'-CCTTAGAGTTTGATGTGGAC-3' (reverse)], Tie-2-specific primers [5'-AAGACCTACGTGAATACCAC-3' (forward) and 5'-GAAACAGAGGGTATACAGATG-3' (reverse)]; and human VEGF 165-specific primers [5'-ACC AAGGCCAGCACATAGG-3' (forward) and 5'-ACGCTC CAGGACTTATACCG-3' (reverse)] (Sigma Genosys Ltd., Cambridge, UK). As a control quantification, s14 mRNA was also subjected to real-time RT-PCR using a set of specific primers [5'-TCCTGCGAGTGCTGTCAGAG -3' (forward) and 5'-TCACCGCCCTACACATCAAA-3' (reverse)] (Sigma Genosys Ltd.). RT-PCRs were performed in a MX3005P system (Stratagene, La Jolla, CA, USA) using Brilliant ${ }^{\circledR}$ SYBR $^{\circledR}$-Green PCR Master Mix (Applied Biosystems, Madrid, Spain) following the manufacturer's instructions. Amplifications were performed for 40 cycles using the following temperature profile: $60^{\circ} \mathrm{C}, 45 \mathrm{sec}$ (annealing); $72^{\circ} \mathrm{C}, 30 \mathrm{sec}$ (extension) and $95^{\circ} \mathrm{C}, 30 \mathrm{sec}$ (denaturation). Each reaction was run 9-fold by quadruplicate. Melting curves were performed to verify that only a single product with no primer-dimers was amplified. For the primers used there were no differences between transcription efficiencies, and the fold-change in each sample was calculated by the $2^{-\Delta \Delta \mathrm{Ct}}$ method (35).

Measurement of ANG-1, ANG-2 and VEGF protein levels. In order to measure ANG-1, ANG-2 and VEGF protein levels in cell co-culture media, samples were collected, centrifuged and processed immediately. For the determination of VEGF concentration in the HUVEC/MCF-7 cell co-culture media a human VEGF Immunoassay kit (R\&D Systems Europe Ltd.) was used. The samples (in triplicate) were processed according to the supplier's instructions. At the end of the procedure, absorbance was determined at a wavelength of $450 \mathrm{~nm}$, with corrections at $540 \mathrm{~nm}$. For the determination of ANG-1 and ANG-2 concentration in the HUVEC/MCF-7 cell co-culture media we used a Human Angiopoietin-1 or -2 Immunoassay kit (R\&D Systems Europe Ltd.) following the supplier's instructions.

Statistical analysis. Data are expressed as the mean \pm standard errors of the mean (SEM). Statistical differences between groups were analyzed using one way analysis of variance (ANOVA), followed by the Student-Newman-Keuls test. Results were considered as statistically significant at $\mathrm{P}<0.05$.

\section{Results}

Melatonin counteracts the stimulatory effect on HUVEC proliferation induced by the presence of tumoral cells. Since reciprocal growth factor exchange between endothelial and breast cancer cells within the tumor microenvironment may directly stimulate neovascularization, we firstly employed co-cultures of HUVECs (lower compartment of the chamber) and MCF-7 (upper compartment of the chamber) cells to investigate whether the presence of malignant epithelial cells affects the growth of the endothelial cells. Indeed, we observed that the presence of breast cancer cells promoted an increase in HUVEC proliferation $(\mathrm{P}<0.01)$ and $1 \mathrm{mM}$ melatonin prevented this stimulatory effect (Fig. 2A). Since melatonin at physiological concentrations did not affect cell proliferation of endothelial cells, we used $1 \mathrm{mM}$ concentration of melatonin in the following experiments. The presence of non-malignant breast epithelial cells in the co-cultures did not promote an increase in HUVEC proliferation and melatonin had no effect (Fig. 2B).

Effects of melatonin on protein levels of angiogenic factors. With the aim of determining whether the increase in HUVEC proliferation could be due to the release of angiogenic factors, such as ANG-1, ANG-2 and VEGF, we measured ANG-1, ANG-2 and VEGF concentrations in the cell co-culture media. The presence of breast cancer cells in the upper compartment of the chamber significantly increased the concentrations of ANG-1, ANG-2 and VEGF $(\mathrm{P}<0.001)$ in the co-culture media, whereas the addition of $1 \mathrm{mM}$ melatonin decreased the concentration of ANG-1, ANG-2 and VEGF and counteracted the stimulatory effect induced by the presence of tumoral cells $(\mathrm{P}<0.001)$ (Fig. 3). 

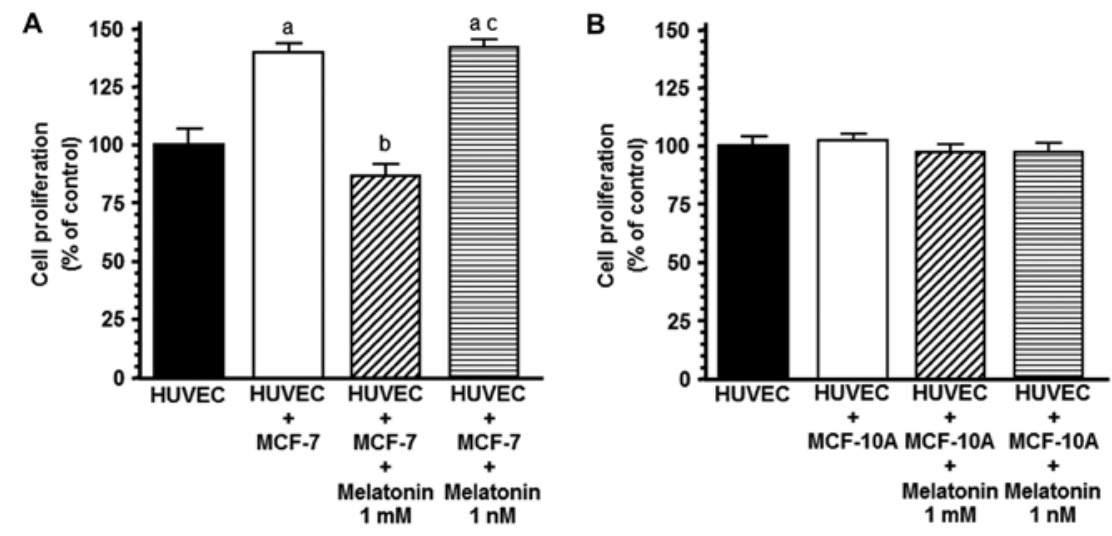

Figure 2. Effects of melatonin (1 mM and $1 \mathrm{nM}$ ) on proliferation of HUVECs, in the presence of (A) breast cancer cells (MCF-7) or (B) non-malignant human mammary epithelial cell line (MCF-10A) in the culture. HUVECs were plated (50x10 ${ }^{4}$ cells/well) on the bottom wells in VCBM supplemented with $2 \%$ FBS and incubated overnight. Then, MCF-7 $\left(40 \times 10^{4}\right)$ or MCF-10A $\left(30 \times 10^{4}\right)$ cells were seeded on the permeable membrane $(0.45 \mu \mathrm{m})$ of the tissue-culture inserts in DMEM supplemented with 10\% FBS for $24 \mathrm{~h}$. Media were then replaced with VCBM supplemented with 2\% FBS containing melatonin (1 mM or $1 \mathrm{nM}$ ) or vehicle (ethanol) for $72 \mathrm{~h}$. At the end of the experiment, cell proliferation was measured by the MTT method. Data are expressed as the percentage of the control group, cultures of only HUVECs (mean \pm SEM). ${ }^{a} \mathrm{P}<0.05$ vs. HUVEC; ${ }^{b} \mathrm{P}<0.001$ vs. HUVEC $+\mathrm{MCF}-7$; ${ }^{\mathrm{c}} \mathrm{P}<0.001$ vs. HUVEC $+\mathrm{MCF}-7+$ melatonin $1 \mathrm{mM}$.
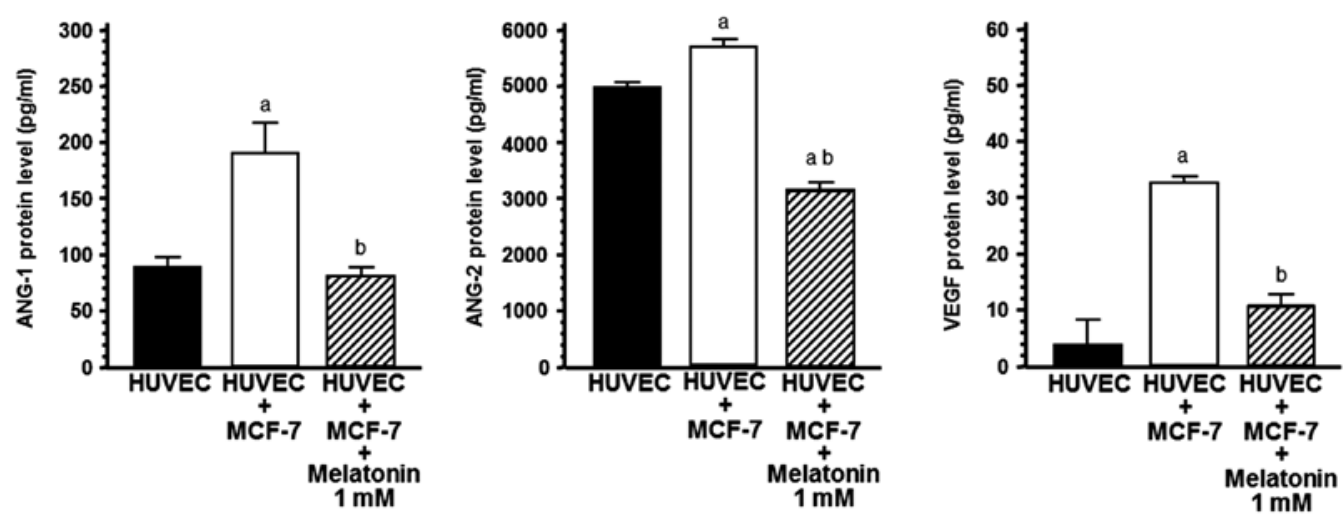

Figure 3. Effects of melatonin (1 mM) on ANG-1, ANG-2 and VEGF protein levels in media of co-cultures of HUVECs and MCF-7 cells determined by ELISA immunoassay kits. HUVECs were plated (50x10/well) on the bottom wells in VCBM supplemented with $2 \%$ FBS and incubated overnight. Then, MCF-7 $\left(40 \times 10^{4}\right)$ cells were seeded on the permeable membrane $(0.45 \mu \mathrm{m})$ of the tissue-culture inserts in DMEM supplemented with $10 \%$ FBS for $24 \mathrm{~h}$. Media were then replaced with VCBM supplemented with $2 \%$ FBS containing melatonin $(1 \mathrm{mM})$ or vehicle (ethanol) for $72 \mathrm{~h}$. At the end of the experiment, media were collected, centrifuged to remove particulates and subjected to measurement of ANG-1, ANG-2 and VEGF protein levels. Data are expressed as the percentage of the control group, cultures of only HUVECs (mean \pm SEM). ${ }^{a} \mathrm{P}<0.001$ vs. HUVEC; ${ }^{\mathrm{b}} \mathrm{P}<0.001$ vs. HUVEC $+\mathrm{MCF}-7$.

Effects of melatonin on $\mathrm{TRNA}$ expression of angiogenic factors. With the aim of determining whether this inhibitory effect of melatonin on ANG-1, ANG-2 and VEGF production was due to a downregulation of ANG-1, ANG-2 and VEGF mRNA expression, total RNA was isolated. RT-PCR was performed using specific primers for human ANG-1, ANG-2 and VEGF and gene s14 as housekeeping. mRNA expression of angiogenic factors in endothelial cells was significantly influenced by co-culture with human breast cancer cells (Fig. 4). ANG-1, ANG-2 and VEGF mRNA expression was significantly $(\mathrm{P}<0.001)$ upregulated during the HUVEC/MCF-7 co-culture relative to the HUVEC monoculture. The addition of melatonin $(1 \mathrm{mM})$ to the co-culture downregulated ANG-1, ANG-2 and VEGF mRNA expression in endothelial cells, showing a $30 \%$ reduction in ANG-1, 50\% downregulation in ANG-2 and 70\% reduction in VEGF mRNA expression (Fig. 4). Melatonin induced a higher decrease in ANG-2 than ANG-1 and shifted ANG-1/ ANG-2 balance in favor of ANG-1. The presence of breast cancer cells also decreased Tie 2 mRNA expression, the specific tyrosine kinase receptor Tie2 of ANG-1 and ANG-2 in endothelial cells. This effect was significantly counteracted by the addition of $1 \mathrm{mM}$ melatonin (Fig. 4).

Significant upregulation of ANG-1, ANG-2 and VEGF mRNA expression occurred also in MCF-7 cells during co-culture with endothelial cells relative to MCF-7 monoculture (Fig. 5). The addition of melatonin $1 \mathrm{mM}$ to the co-culture significantly $(\mathrm{P}<0.001)$ downregulated ANG-1, ANG-2 and VEGF mRNA expression and upregulated Tie2 mRNA expression in breast cancer cells (Fig. 5).

The expression of the angiogenic factors in endothelial cells, in the presence of non-malignant MCF-10A breast epithelial cell line was also assessed. ANG-1, ANG-2, Tie2 and VEGF mRNA expression levels were not modified during the HUVEC/MCF-10A co-culture in comparison to the HUVEC monoculture (Fig. 6). The addition of melatonin $(1 \mathrm{mM})$ to the co-culture only upregulated ANG-2 mRNA expression in the endothelial cells (Fig. 6). 

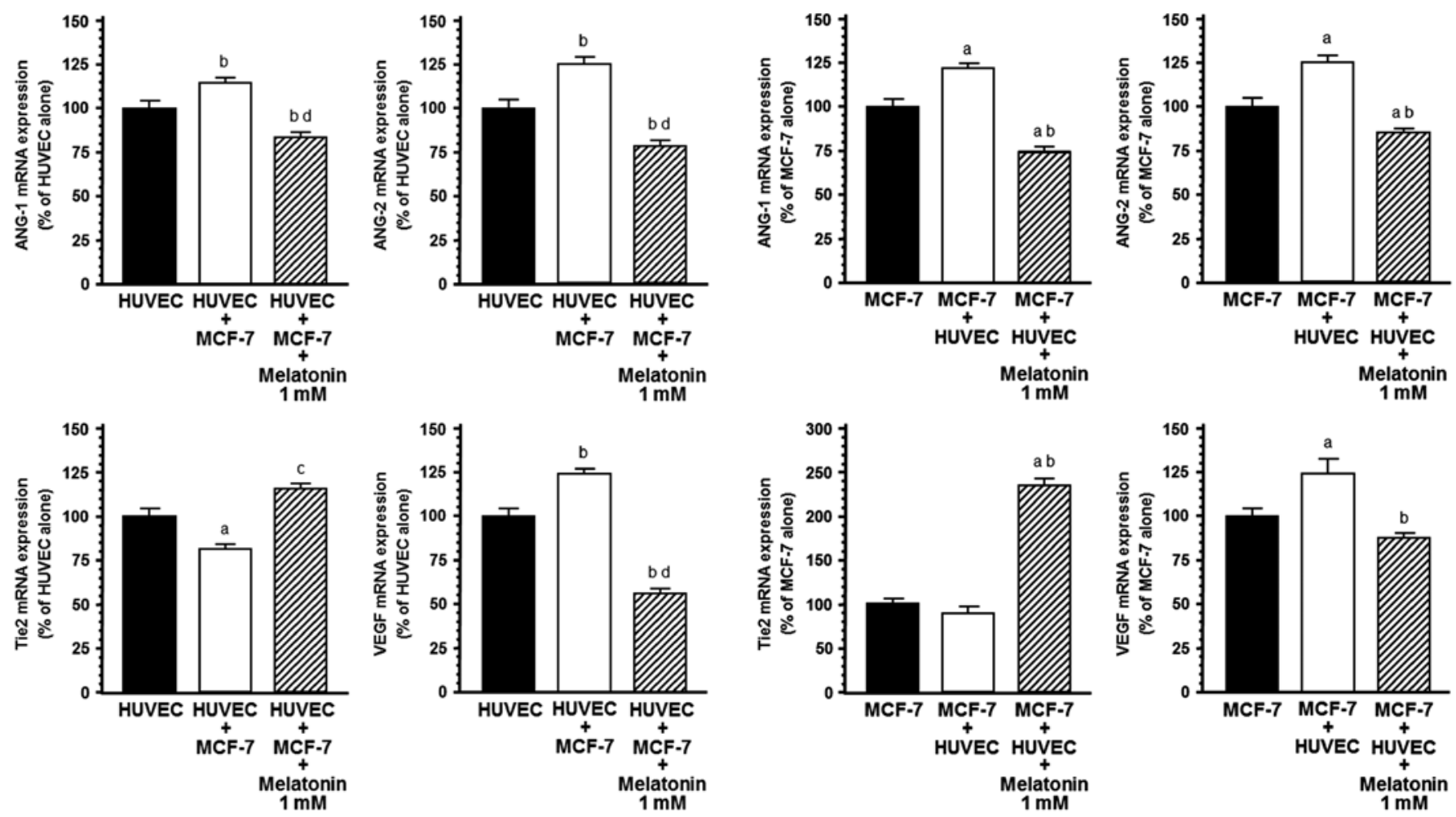

Figure 4. Effects of melatonin (1 mM) on ANG-1, ANG-2, Tie2 and VEGF mRNA expression in HUVEC co-culture with MCF-7 cells. HUVECs were plated $\left(50 \times 10^{4} /\right.$ well) on the bottom wells in VCBM supplemented with $2 \%$ FBS and incubated overnight. Then, MCF-7 $\left(40 \times 10^{4}\right)$ cells were seeded on the permeable membrane $(0.45 \mu \mathrm{m})$ of the tissue-culture inserts in DMEM supplemented with $10 \%$ FBS for $24 \mathrm{~h}$. Media were then replaced with VCBM supplemented with $2 \%$ FBS containing melatonin $(1 \mathrm{mM})$ or vehicle (ethanol) for $4 \mathrm{~h}$. Total mRNA was isolated from cells and reverse transcribed cDNA was subjected to RT-PCR using specific primers for ANG-1, ANG-2, Tie2, VEGF or s14. Data are expressed as the percentage of the control group, cultures of only HUVECs (mean \pm SEM). ${ }^{a} \mathrm{P}<0.05$ vs. HUVEC; ${ }^{b} \mathrm{P}<0.001$ vs. HUVEC; ${ }^{\circ} \mathrm{P}<0.05$ vs. HUVEC + MCF-7; ${ }^{\mathrm{d}} \mathrm{P}<0.001$ vs. HUVEC + MCF-7.

Estradiol $(10 \mathrm{nM})$, added to both compartments of the multi-well plate, increased ANG-1, ANG-2 and VEGF mRNA expression in HUVECs and melatonin $(1 \mathrm{mM})$ significantly $(\mathrm{P}<0.001)$ counteracted this effect (Fig. 7).

\section{Discussion}

Tumor vascular neo-angiogenesis is an intrincate dynamic process that has an important role in tumor ontogenesis and progression. The VEGF pathway and more recently, ANG/Tie2 receptor signaling are considered the main regulators of different mechanisms of tumor vascularization (4,9-12). VEGF synthesized in and secreted by cancer cells, plays a crucial role in the progression and development of malignant mammary tumors since VEGF stimulates vascular permeability and proliferation of endothelial cells from contiguous blood vessels (36). Angiopoietin-1 (ANG-1) maintains the integrity of vasculature and is expressed in perivascular cells such as pericytes, vascular smooth muscle cells, fibroblasts and tumor cells, whereas ANG-2 is mainly released by endothelial cells only at the sites of vascular remodeling. Both ANG-1 and ANG-2 are ligands of the Tie2 tyrosine kinase receptor presenting similar affinities but antagonistic effects. The

Figure 5. Effects of melatonin (1 mM) on ANG-1, ANG-2, Tie2 and VEGF mRNA expression in MCF-7 co-culture with HUVECs. MCF-7 cells were plated $\left(80 \times 10^{4} /\right.$ well) on the bottom wells in DMEM supplemented with $10 \%$ FBS and incubated overnight. Then, HUVECs $\left(30 \times 10^{4}\right)$ were seeded on the permeable membrane $(0.45 \mu \mathrm{m})$ of the tissue-culture inserts in VCBM supplemented with $2 \%$ FBS for $24 \mathrm{~h}$. Media were then replaced with VCBM supplemented with $2 \%$ FBS containing melatonin $(1 \mathrm{mM})$ or vehicle (ethanol) for $4 \mathrm{~h}$. Total mRNA was isolated from cells and reverse transcribed. cDNA was subjected to RT-PCR using specific primers for ANG-1, ANG-2, VEGF, Tie2 or s14. Data are expressed as the percentage of the control group, cultures of only MCF-7 cells (mean $\pm \mathrm{SEM}$ ). ${ }^{\mathrm{a}} \mathrm{P}<0.001 \mathrm{vs}$. HUVEC; ${ }^{\mathrm{b}} \mathrm{P}<0.001$ vs. HUVEC + MCF-7.

binding of ANG-1 triggers a signal that finally induces vessel maturation and stabilizes tumor vasculature. However, ANG-2 competes with ANG-1 for Tie2 binding, causing vessel regression in the absence of angiogenic factors, such as VEGF, whereas it promotes angiogenesis in the presence of $\operatorname{VEGF}(9,10,37,38)$. Thus, the ratio of ANG-1 to ANG-2 is critical in balancing Tie2 signaling pathways and in regulating vascular homeostasis. Angiopoietins seem to act in a complementary and coordinated manner with VEGF, playing a later role in vascular development.

Melatonin exerts oncostatic effects through different biological mechanisms (39-41). The first description of the antiangiogenic properties of melatonin came from a clinical study showing a decline in serum levels of VEGF in cancer patients treated with this indoleamine (42). More recently it has been demonstrated that melatonin exerts antiangiogenic actions mainly through its inhibitory actions on VEGF expression and protein levels $(28,29,43,44)$. The aim of the present study was to study whether melatonin may modulate in a coordinated action the production of ANG-1 and ANG-2, their cognate Tie2 receptor and VEGF in co-cultures of human endothelial and breast cancer cells. 

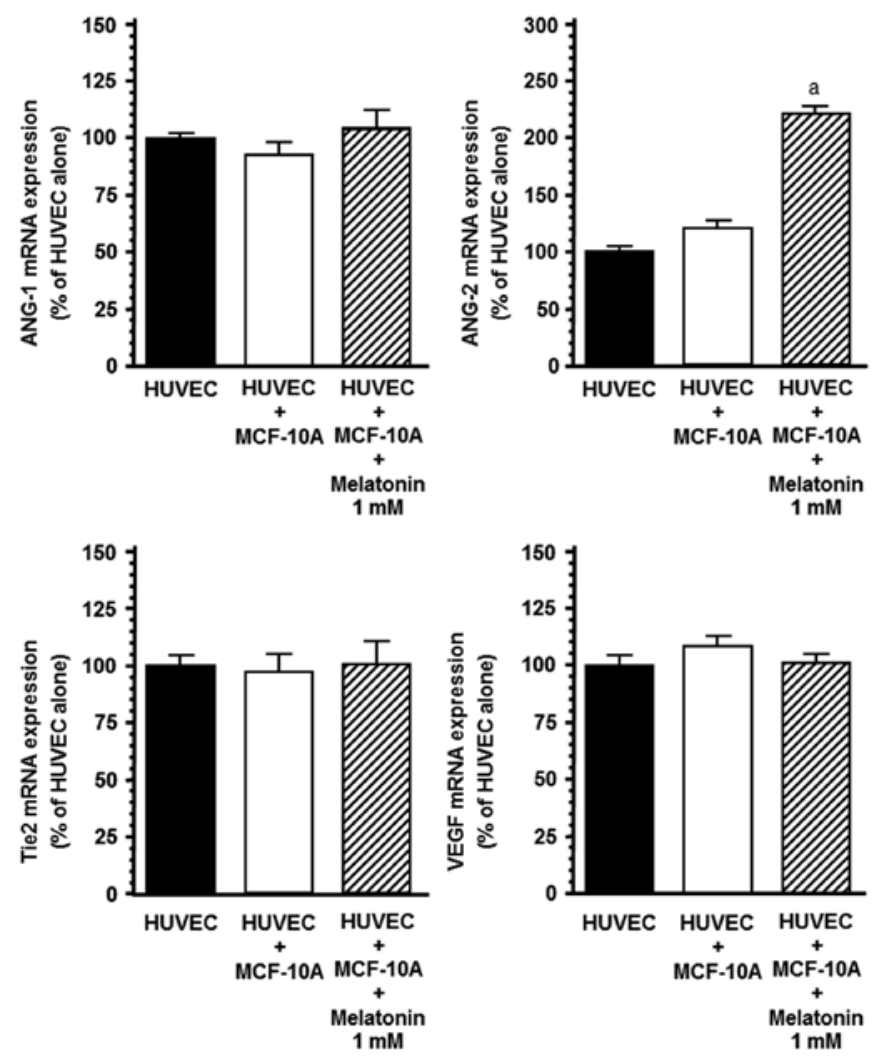

Figure 6. Effects of melatonin (1 mM) on ANG-1, ANG-2, Tie2 and VEGF mRNA expression in the HUVEC co-culture with MCF-10A cells. HUVECs were plated $\left(50 \times 10^{4} /\right.$ well) on the bottom wells in VCBM supplemented with $2 \%$ FBS and incubated overnight. Then, MCF-10A $\left(30 \times 10^{4}\right)$ cells were seeded on the permeable membrane $(0.45 \mu \mathrm{m})$ of the tissue-culture inserts in DMEM/F12 supplemented with $5 \%$ horse serum, $0.5 \mu \mathrm{g} / \mathrm{ml}$ hydrocortisone, $20 \mathrm{ng} / \mathrm{ml}$ epidermal growth factor, $100 \mathrm{ng} / \mathrm{ml}$ cholera toxin and $10 \mu \mathrm{g} / \mathrm{ml}$ insulin for $24 \mathrm{~h}$. Media were then replaced with VCBM supplemented with $2 \%$ FBS containing melatonin $(1 \mathrm{mM})$ or vehicle (ethanol) for $4 \mathrm{~h}$. Total mRNA was isolated from cells and reverse transcribed. cDNA was subjected to RT-PCR using specific primers for ANG-1, ANG-2, VEGF, Tier or s14. Data are expressed as the percentage of the control group, cultures of only HUVECs (mean \pm SEM). ${ }^{a} \mathrm{P}<0.001$ vs. other groups.

Our data, firstly, demonstrated that human breast cancer cells can exert a potent influence on endothelial cells and vice versa. The presence of breast cancer cells in the co-cultures promoted an increase in HUVEC proliferation as well as an upregulation of ANG-1, ANG-2 and VEGF mRNA expression in endothelial cells in comparison to the HUVEC monocultures. Additionally, the presence of tumor cells also induced the downregulation of Tie2 mRNA expression in endothelial cells. This pro-angiogenic response was not observed in co-cultures of endothelial and non-malignant breast epithelial cells which highlights an important difference in the reciprocal interactions between endothelial and malignant and nonmalignant breast epithelial cells. The addition of melatonin at pharmacological concentrations $(1 \mathrm{mM})$ to the co-culture downregulated ANG-1, ANG-2 and VEGF mRNA expression in endothelial cells and counteracted the reduction in Tie2 mRNA expression induced by the presence of the tumor cells. Melatonin shifted the ANG-1/ANG-2 balance in favor of ANG-1, since it induced a higher reduction in ANG-2 than ANG-1 expression. In addition, the presence of breast cancer cells significantly increased the levels of ANG-1, ANG-2 and
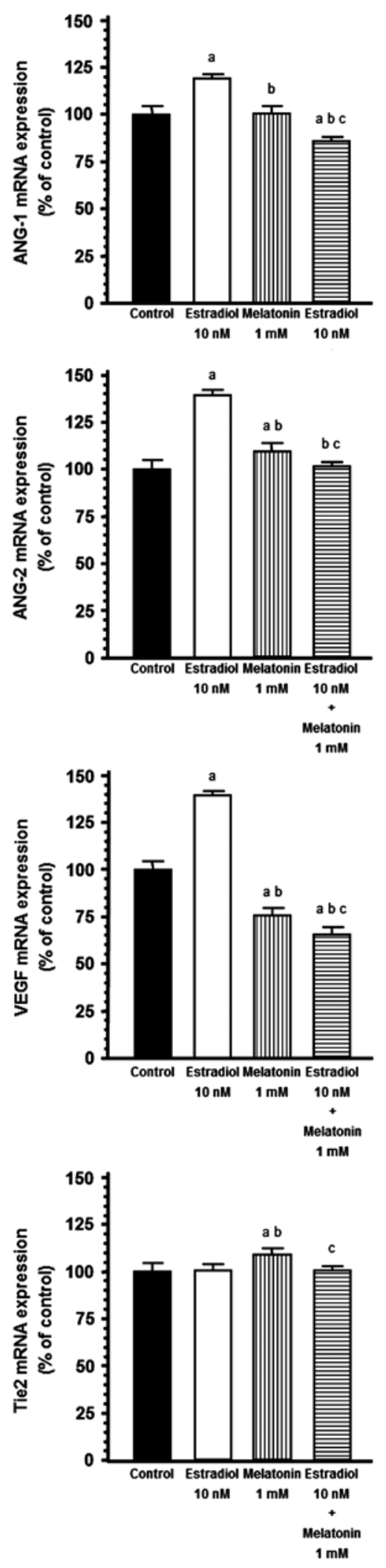

Figure 7. Effects of estradiol (10 $\mathrm{nM})$ and melatonin (1 mM) on ANG-1, ANG-2, VEGF and Tie2 mRNA expression in HUVECs. HUVECs were seeded (50x104/plate) into Falcon 6-multiwell plates, in VCBM supplemented with $2 \%$ FBS for $24 \mathrm{~h}$. Media were then replaced with VCBM supplemented with $2 \%$ FBS containing melatonin $(1 \mathrm{mM})$ and/or estradiol $(10 \mathrm{nM})$ or vehicle (ethanol) for $4 \mathrm{~h}$. Total mRNA was isolated from cells and reverse transcribed. cDNA was subjected to RT-PCR using specific primers for ANG-1, ANG-2, VEGF, Tie2 or s14. Data are expressed as the percentage of the control group, cultures of only HUVECs (mean $\pm \mathrm{SEM}$ ). ${ }^{\mathrm{a}} \mathrm{P}<0.001$ vs. HUVEC; ${ }^{\mathrm{b}} \mathrm{P}<0.001$ vs. HUVEC + estradiol $10 \mathrm{nM} ;{ }^{\circ} \mathrm{P}<0.001$ vs. HUVEC + melatonin $1 \mathrm{mM}$. 
VEGF in the co-culture media, whereas the addition of $1 \mathrm{mM}$ melatonin decreased the concentration of ANG-1, ANG-2 and VEGF and counteracted the stimulatory effect triggered by the presence of tumoral cells. The greatest melatonin inhibition of endothelial cell proliferation was found with melatonin at a concentration of $1 \mathrm{mM}$ as previously demonstrated (43). The oncostatic effects of melatonin on different cells have been related with factors such as the concentration of melatonin in the cultures, the time of exposure to this indolamine and the concrete characteristics of the cells studied. In our study, only pharmacological concentrations of melatonin had an inhibitory effect on HUVEC proliferation. A potent inhibitory action of melatonin at nanomolar concentrations on human breast cancer cell proliferation has been previously shown $(15,16,41,45)$. However, melatonin at high doses is required to obtain antitumoral effects in other types of normal cells and tumor cells $(43,46-50)$. It has been described that the melatonin concentration in the cerebrospinal fluid is higher than that in blood since melatonin is a highly lipophilic molecule which may easily cross the blood-brain barrier (51). Additionally, it is known that melatonin can become at least one thousand times more concentrated in tumoral and adipose tissues of the breast (52). The fact that melatonin achieves high concentrations in some tissues may justify why high levels of melatonin are necessary to obtain some antitumoral effects of this indolamine. Thus, we used this pharmacological concentration of melatonin throughout our experimental study, since only this dose of melatonin was effective in inhibiting the proliferation of HUVECs.

Our results also demonstrated that the pro-angiogenic activity of breast cancer cells, but not non-malignant mammary epithelial cells, was significantly enhanced by the presence of endothelial cells. The presence of endothelial cells upregulated ANG-1, ANG-2, Tie2 and VEGF mRNA expression in the MCF-7 cells and the addition of melatonin $1 \mathrm{mM}$ to the co-culture significantly downregulated ANG-1, ANG-2 and VEGF mRNA expression and upregulated Tie 2 mRNA expression in the breast cancer cells. It has been described that the angiopoietin-Tie2 system has an autoregulation feedback system that modulates the overall activity of the Tie 2 system. ANG-1, but not ANG-2, downregulates Tie2 mRNA expression (53). In our co-culture experimental design, melatonin downregulated both ANG-1 and ANG-2 mRNA expression. The lower levels of ANG-1 may explain the upregulation of Tie 2 mRNA expression induced by melatonin.

Since it is known that estrogens modulate angiogenesis and little information is yet available regarding the influence of estrogens on angiopoietins, we also aimed to study the effects of estradiol on angiopoietin and VEGF mRNA expression in endothelial cells with and without melatonin. Estradiol (10 $\mathrm{nM}$ ) increased ANG-1, ANG-2 and VEGF mRNA expression and melatonin $(1 \mathrm{mM})$ significantly counteracted this effect. Melatonin is well known for its oncostatic effects on estrogen-dependent breast tumors mainly by two antiestrogenic mechanisms: interfering with estrogen signaling pathways at the estrogen receptor level $(17,19,20)$ and regulating both the activity and expression of enzymes involved in local estrogen biosynthesis in tumor cells and peritumoral fibroblasts $(40,41,45)$. This inhibitory action of melatonin counteracting the effect of estrogens on angiopoietin and
VEGF expression, could be included in the oncostatic actions of melatonin interfering at different levels in estrogen signaling pathways. There has been increasing evidence that estrogens regulate angiopoietin expression; however, the differential influence of estrogens on ANG-1 and ANG-2 mRNA expression varies considerably between studies. In non-reproductive rat tissues, estradiol increased ANG-2 mRNA expression whereas it reduced ANG-1 mRNA expression (54). There is one study reporting an inverse correlation of ANG-1 mRNA expression with the level of $\mathrm{ER} \alpha$ in breast cancer cell lines (55). In our cultures, estradiol stimulated endothelial growth and increased ANG-1, ANG-2 and VEGF mRNA expression while melatonin decreased ANG-1, ANG-2 and VEGF mRNA expression and increased Tie 2 mRNA expression. The overexpression of Tie 2 induced by melatonin in endothelial cells may lead to an increased vessel stabilization, thereby making the vasculature less susceptible to pro-angiogenic factors such as VEGF.

Sequential and complementary expression of ANG-1, ANG-2 and VEGF has been described as crucial for successful angiogenesis. Therefore, any interruption or disturbance in this balanced expression may significantly affect the angiogenic process. In the presence of VEGF, ANG-2 induces vascular sprouting and disrupts the interaction between pericytes and endothelial cells, promoting the destabilization of blood vessels and then increasing VEGF stimulation. In contrast, in the absence of VEGF, ANG-2 works as a suppressor that potentiates vessel regression (37). Moreover, systemic expression of ANG-2 has been described to increase metastasis and specific blockade of ANG-2 reduces metastasis development (56). In preclinical studies, it was demonstrated that the association of ANG-2 blockade with VEGF blockade and with cytotoxic drugs produced significantly greater inhibitory actions on tumor growth and angiogenesis than any agent alone (57). Moreover, inhibition of ANG-2 or VEGF reduced tumor growth but the inhibition of both together slowed tumor growth even more and decreased the number of endothelial sprouts to a degree greater than either inhibitor alone (58). The use of ANG-2 blockers reduced vessel sprouting, while anti-VEGF antibodies that work as blockers of VEGF function caused vessel regression (58). Thus, high ANG-2 levels may interfere with the efficacy of anti-VEGF therapy. In studies with mice, the use of a specific anti-ANG-2 monoclonal antibody reduced lung metastasis and lung lymph node metastasis from a non-small cell carcinoma (59) and decreased metastasis in spontaneous breast carcinoma, which may be explained at least to a certain degree by reducing the pro-angiogenic action of monocytes associated to tumors $(59,60)$. Recent studies suggest the benefits of antitumoral treatments that target multiple antiangiogenic pathways, by acting on different receptor tyrosine kinases, with the purpose of impairing tumor neovascularization more efficiently than either inhibitor alone (58). The fact that melatonin has complementary actions and coordinates at the same time a downregulation of angiopoietins with a reduction in VEGF could be an effective therapeutic strategy for blocking tumor angiogenesis and growth.

The present study was the first to demonstrate the effect of melatonin on angiopoietins in human breast cancer and endothelial cells. We found that the presence of breast cancer cells increased endothelial cell proliferation and $1 \mathrm{mM}$ melatonin 
prevented this effect. ANG-1, ANG-2 and VEGF levels in co-culture media and mRNA expression were upregulated and Tie2 mRNA expression was downregulated in HUVECs and MCF-7 cells. Melatonin (1 mM) downregulated ANG-1, ANG-2 and VEGF levels in co-culture media and mRNA expression in both types of cells and upregulated Tie2 mRNA expression in HUVECs. ANG-1, ANG-2, Tie2 and VEGF mRNA expression were not modified during HUVEC/ MCF-10A co-culture. Estradiol (10 nM) increased ANG-1, ANG-2 and VEGF mRNA expression in HUVECs and melatonin $(1 \mathrm{mM})$ counteracted this effect. Our findings suggest that melatonin simultaneously coordinates downregulation of angiopoietins with a reduction of VEGF which could be an important action for blocking tumor angiogenesis. Further experiments are necessary to clarify the mechanisms involved in the antiangiogenic action of melatonin.

\section{Acknowledgements}

The present study was supported by grants from the Spanish Economy and Competitiveness Ministry (SAF2013-42012-P, SAF2016-77103-P), and from the Instituto de Investigación Sanitaria Valdecilla (IDIVAL) (APG/12).

\section{References}

1. Bergers $\mathrm{G}$ and Benjamin LE: Tumorigenesis and the angiogenic switch. Nat Rev Cancer 3: 401-410, 2003.

2. Bareschino MA, Schettino C, Colantuoni G, Rossi E, Rossi A, Maione P, Ciardiello F and Gridelli C: The role of antiangiogenetic agents in the treatment of breast cancer. Curr Med Chem 18: 5022-5032, 2011.

3. Cook KM and Figg WD: Angiogenesis inhibitors: Current strategies and future prospects. CA Cancer J Clin 60: 222-243, 2010.

4. Danza K, Pilato B, Lacalamita R, Addati T, Giotta F, Bruno A, Paradiso A and Tommasi S: Angiogenetic axis angiopoietins/Tie2 and VEGF in familial breast cancer. Eur J Hum Genet 21: 824-830, 2013.

5. Senger DR, Van de Water L, Brown LF, Nagy JA, Yeo KT, Yeo TK, Berse B, Jackman RW, Dvorak AM and Dvorak HF: Vascular permeability factor (VPF, VEGF) in tumor biology. Cancer Metastasis Rev 12: 303-324, 1993.

6. Gu Q, Wang D, Wang X, Peng R, Liu J, Jiang T, Wang Z, Wang S and Deng $\mathrm{H}$ : Basic fibroblast growth factor inhibits radiationinduced apoptosis of HUVECs. I. The PI3K/AKT pathway and induction of phosphorylation of BAD. Radiat Res 161: 692-702, 2004.

7. Gingis-Velitski S, Zetser A, Flugelman MY, Vlodavsky I and Ilan N: Heparanase induces endothelial cell migration via protein kinase B/Akt activation. J Biol Chem 279: 23536-23541, 2004.

8. Gerber HP, McMurtrey A, Kowalski J, Yan M, Keyt BA, Dixit V and Ferrara N: Vascular endothelial growth factor regulates endothelial cell survival through the phosphatidylinositol3'-kinase/Akt signal transduction pathway. Requirement for Flk-1/KDR activation. J Biol Chem 273: 30336-30343, 1998.

9. Fagiani E and Christofori G: Angiopoietins in angiogenesis. Cancer Lett 328: 18-26, 2013.

10. Cao Y, Sonveaux P, Liu S, Zhao Y, Mi J, Clary BM, Li CY, Kontos CD and Dewhirst MW: Systemic overexpression of angiopoietin-2 promotes tumor microvessel regression and inhibits angiogenesis and tumor growth. Cancer Res 67: 3835-3844, 2007.

11. Thomas M and Augustin HG: The role of the Angiopoietins in vascular morphogenesis. Angiogenesis 12: 125-137, 2009.

12. Tait CR and Jones PF: Angiopoietins in tumours: The angiogenic switch. J Pathol 204: 1-10, 2004.

13. Thurston $G$ and Daly $C$ : The complex role of angiopoietin-2 in the angiopoietin-tie signaling pathway. Cold Spring Harb Perspect Med 2: a006550, 2012.

14. Bhadada SV, Goyal BR and Patel MM: Angiogenic targets for potential disorders. Fundam Clin Pharmacol 25: 29-47, 2011.
15. Hill SM and Blask DE: Effects of the pineal hormone melatonin on the proliferation and morphological characteristics of human breast cancer cells (MCF-7) in culture. Cancer Res 48: 6121-6126, 1988.

16. Cos S and Sánchez-Barceló EJ: Melatonin and mammary pathological growth. Front Neuroendocrinol 21: 133-170, 2000.

17. Blask DE, Sauer LA and Dauchy RT: Melatonin as a chronobiotic/anticancer agent: Cellular, biochemical, and molecular mechanisms of action and their implications for circadian-based cancer therapy. Curr Top Med Chem 2: 113-132, 2002.

18. Reiter RJ: The pineal and its hormones in the control of reproduction in mammals. Endocr Rev 1: 109-131, 1980.

19. Molis TM, Spriggs LL and Hill SM: Modulation of estrogen receptor mRNA expression by melatonin in MCF-7 human breast cancer cells. Mol Endocrinol 8: 1681-1690, 1994.

20. Cos S, Blask DE, Lemus-Wilson A and Hill AB: Effects of melatonin on the cell cycle kinetics and 'estrogen-rescue' of MCF-7 human breast cancer cells in culture. J Pineal Res 10: 36-42, 1991.

21. Allegra M, Reiter RJ, Tan DX, Gentile C, Tesoriere L and Livrea MA: The chemistry of melatonin's interaction with reactive species. J Pineal Res 34: 1-10, 2003.

22. Fraschini F, Demartini G, Esposti D and Scaglione F: Melatonin involvement in immunity and cancer. Biol Signals Recept 7: 61-72, 1998.

23. Leon-Blanco MM, Guerrero JM, Reiter RJ, Calvo JR and Pozo D: Melatonin inhibits telomerase activity in the MCF-7 tumor cell line both in vivo and in vitro. J Pineal Res 35: 204-211, 2003.

24. Martínez-Campa CM, Alonso-González C, Mediavilla MD, Cos S, González A and Sanchez-Barcelo EJ: Melatonin downregulates hTERT expression induced by either natural estrogens (17beta-estradiol) or metalloestrogens (cadmium) in MCF-7 human breast cancer cells. Cancer Lett 268: 272-277, 2008.

25. Blask DE, Dauchy RT, Sauer LA, Krause JA and Brainard GC: Growth and fatty acid metabolism of human breast cancer (MCF-7) xenografts in nude rats: Impact of constant light-induced nocturnal melatonin suppression. Breast Cancer Res Treat 79: 313-320, 2003

26. Blask DE, Dauchy RT and Sauer LA: Putting cancer to sleep at night: The neuroendocrine/circadian melatonin signal. Endocrine 27: 179-188, 2005

27. Alvarez-García V, González A, Martínez-Campa C, AlonsoGonzález C and Cos S: Melatonin modulates aromatase activity and expression in endothelial cells. Oncol Rep 29: 2058-2064, 2013.

28. Alvarez-García V, González A, Alonso-González C, Martínez-Campa $\mathrm{C}$ and Cos S: Regulation of vascular endothelial growth factor by melatonin in human breast cancer cells. J Pineal Res 54: 373-380, 2013.

29. Alvarez-García V, González A, Alonso-González C, MartínezCampa $\mathrm{C}$ and Cos S: Antiangiogenic effects of melatonin in endothelial cell cultures. Microvasc Res 87: 25-33, 2013.

30. Cos S, Alvarez-García V, González A, Alonso-González C and Martínez-Campa C: Melatonin modulation of crosstalk among malignant epithelial, endothelial and adipose cells in breast cancer (Review). Oncol Lett 8: 487-492, 2014.

31. Kajdaniuk D, Marek B, Kos-Kudła B, Zwirska-Korczala K, Ostrowska Z, Buntner B and Szymszal J: Does the negative correlation found in breast cancer patients between plasma melatonin and insulin-like growth factor-I concentrations imply the existence of an additional mechanism of oncostatic melatonin influence involved in defense? Med Sci Monit 8: CR457-CR461, 2002.

32. Fandrey $\mathbf{J}$ and Genius $\mathbf{J}$ : Reactive oxygen species as regulators of oxygen dependent gene expression. Adv Exp Med Biol 475: $153-159,2000$

33. Lobov IB, Brooks PC and Lang RA: Angiopoietin-2 displays VEGF-dependent modulation of capillary structure and endothelial cell survival in vivo. Proc Natl Acad Sci USA 99: 11205-11210, 2002.

34. Mosmann T: Rapid colorimetric assay for cellular growth and survival: Application to proliferation and cytotoxicity assays. J Immunol Methods 65: 55-63, 1983.

35. Livak KJ and Schmittgen TD: Analysis of relative gene expression data using real-time quantitative PCR and the $2^{-\Delta \Delta C_{\mathrm{T}}}$ method. Methods 25: 402-408, 2001.

36. Liang Y and Hyder SM: Proliferation of endothelial and tumor epithelial cells by progestin-induced vascular endothelial growth factor from human breast cancer cells: Paracrine and autocrine effects. Endocrinology 146: 3632-3641, 2005. 
37. Holash J, Wiegand SJ and Yancopoulos GD: New model of tumor angiogenesis: Dynamic balance between vessel regression and growth mediated by angiopoietins and VEGF. Oncogene 18: 5356-5362, 1999.

38. Currie MJ, Gunningham SP, Han C, Scott PA, Robinson BA, Harris AL and Fox SB: Angiopoietin-1 is inversely related to thymidine phosphorylase expression in human breast cancer, indicating a role in vascular remodeling. Clin Cancer Res 7: 918-927, 2001.

39. Sánchez-Barceló EJ, Cos S, Fernández R and Mediavilla MD: Melatonin and mammary cancer: A short review. Endocr Relat Cancer 10: 153-159, 2003.

40. Cos S, González A, Martínez-Campa C, Mediavilla MD, Alonso-González C and Sánchez-Barceló EJ: Estrogen-signaling pathway: A link between breast cancer and melatonin oncostatic actions. Cancer Detect Prev 30: 118-128, 2006.

41. Cos S, González A, Martínez-Campa C, Mediavilla MD, AlonsoGonzález C and Sánchez-Barceló EJ: Melatonin as a selective estrogen enzyme modulator. Curr Cancer Drug Targets 8 : 691-702, 2008.

42. Lissoni P, Rovelli F, Malugani F, Bucovec R, Conti A and Maestroni GJ: Anti-angiogenic activity of melatonin in advanced cancer patients. Neuro Endocrinol Lett 22: 45-47, 2001.

43. Cui P, Luo Z, Zhang H, Su Y, Li A, Li H, Zhang J, Yang Z and Xiu R: Effect and mechanism of melatonin's action on the proliferation of human umbilical vein endothelial cells. J Pineal Res 41: 358-362, 2006.

44. Dai M, Cui P, Yu M, Han J, Li H and Xiu R: Melatonin modulates the expression of VEGF and HIF- $1 \alpha$ induced by $\mathrm{CoCl}_{2}$ in cultured cancer cells. J Pineal Res 44: 121-126, 2008.

45. Cos S, Martínez-Campa C, Mediavilla MD and SánchezBarceló EJ: Melatonin modulates aromatase activity in MCF-7 human breast cancer cells. J Pineal Res 38: 136-142, 2005.

46. Alvarez-García V, González A, Alonso-González C, MartínezCampa C and Cos S: Melatonin interferes in the desmoplastic reaction in breast cancer by regulating cytokine production. J Pineal Res 52: 282-290, 2012.

47. González A, Alvarez-García V, Martínez-Campa C, AlonsoGonzález $\mathrm{C}$ and Cos S: Melatonin promotes differentiation of 3T3-L1 fibroblasts. J Pineal Res 52: 12-20, 2012.

48. Cui P, Yu M,Luo Z, Dai M, Han J, Xiu R and Yang Z: Intracellular signaling pathways involved in cell growth inhibition of human umbilical vein endothelial cells by melatonin. J Pineal Res 44 107-114, 2008

49. García-Santos G, Antolín I, Herrera F, Martín V, RodríguezBlanco J, del Pilar Carrera M and Rodríguez C: Melatonin induces apoptosis in human neuroblastoma cancer cells. J Pineal Res 41: 130-135, 2006.
50. Sainz RM, Mayo JC, Tan DX, León J, Manchester L and Reiter RJ: Melatonin reduces prostate cancer cell growth leading to neuroendocrine differentiation via a receptor and PKA independent mechanism. Prostate 63: 29-43, 2005.

51. Longatti P, Perin A, Rizzo V, Comai S, Giusti P and Costa CV: Ventricular cerebrospinal fluid melatonin concentrations investigated with an endoscopic technique. J Pineal Res 42: 113-118, 2007.

52. Maestroni GJ and Conti A: Melatonin in human breast cancer tissue: Association with nuclear grade and estrogen receptor status. Lab Invest 75: 557-561, 1996.

53. Hashimoto T, Wu Y, Boudreau N, Li J, Matsumoto M and Young W: Regulation of tie 2 expression by angiopoietin - potential feedback system. Endothelium 11: 207-210, 2004.

54. Ye F, Florian M, Magder SA and Hussain SN: Regulation of angiopoietin and Tie-2 receptor expression in non-reproductive tissues by estrogen. Steroids 67: 305-310, 2002.

55. Harfouche R, Echavarria R, Rabbani SA, Arakelian A, Hussein MA and Hussain SN: Estradiol-dependent regulation of angiopoietin expression in breast cancer cells. J Steroid Biochem Mol Biol 123: 17-24, 2011.

56. Holopainen T, Saharinen P, D'Amico G, Lampinen A, Eklund L, Sormunen R, Anisimov A, Zarkada G, Lohela M, Heloterä H, et al: Effects of angiopoietin-2-blocking antibody on endothelial cell-cell junctions and lung metastasis. J Natl Cancer Inst 104: 461-475, 2012.

57. Brown JL, Cao ZA, Pinzon-Ortiz M, Kendrew J, Reimer C, Wen S, Zhou JQ, Tabrizi M, Emery S, McDermott B, et al: A human monoclonal anti-ANG2 antibody leads to broad antitumor activity in combination with VEGF inhibitors and chemotherapy agents in preclinical models. Mol Cancer Ther 9: 145-156, 2010.

58. Hashizume H, Falcón BL, Kuroda T, Baluk P, Coxon A, Yu D, Bready JV, Oliner JD and McDonald DM: Complementary actions of inhibitors of angiopoietin-2 and VEGF on tumor angiogenesis and growth. Cancer Res 70: 2213-2223, 2010.

59. Leow CC, Coffman K, Inigo I, Breen S, Czapiga M, Soukharev S, Gingles N, Peterson N, Fazenbaker C, Woods R, et al: MEDI3617, a human anti-Angiopoietin 2 monoclonal antibody, inhibits angiogenesis and tumor growth in human tumor xenograft models. Int J Oncol 40: 1321-1330, 2012.

60. Mazzieri R, Pucci F, Moi D, Zonari E, Ranghetti A, Berti A, Politi LS, Gentner B, Brown JL, Naldini L, et al: Targeting the ANG2/TIE2 axis inhibits tumor growth and metastasis by impairing angiogenesis and disabling rebounds of proangiogenic myeloid cells. Cancer Cell 19: 512-526, 2011. 